decrease of beta globulin. Hemoconcentration was not dominant. These could be the causative adjuncts to the microcirculatory insufficiency within the brain and upper cord.

In the dynamo-vertebral and myotonic cerebrovascular insufficiency, stellate ganglion-block or perivertebral infiltration block and epidural infusion block might be effective. In the cases of microcirculatory insufficiency, administration of low molecular weight dextran or other plasma expanders is to be effective. Besides, adequate regulation of fibrinolytic activation system should be effective. Indication of operation must be expanded.

\title{
k-21. Effect of Stellate Ganglion Block upon Carotid and Vertebral Blood Flow in Dogs and in a Man
}

\author{
Shunshiro Kondo, Kazuaki SugIUra, Kiyoshi OHWakI and Yoshinori Yabe \\ Department of Surgery, Nippon Medical School
}

In previous papers we demonstrated that so-called whiplash injury becomes manifest when an autonomic nervous system loses its normal balance. We have treated 110 whiplash injury patients in three years with a course of stellate ganglion block which has proved to be quite satisfactory.

Stellate ganglion block was employed by many clinicians for the treatment of various cerebrovascular diseases since a known report by Leriche. Forbes and Cobb proved experimentally the regulatory effects of cervical sympathetic system upon intracranial blood flow by microscopic observation of pia-arachnoid blood vessels before and after electric stimulation of the cervical sympathetic chain.

The works of Gibbs, Lennox, Harmel, and Scheinberg were contrary to the results obtained by aforementioned workers. They could find no remarkable changes in cerebral blood flow after stellate ganglion block, while Shenkin, measuring by nitrous oxide technique, suggested an increase of flow after bilateral stellate ganglionectomy.

We studied common carotid and vertebral blood flow in mongrel dogs and internal carotid flow in one patient before and after stellate ganglion block using electromagnetic flowmeter. We knew that it causes relatively constant increase of flow in 20 out of 22 dogs examined. The increase are $12.8 \%$ in the common carotid and $35.6 \%$ in the vertebral arteries in the average. We have also ascertained that these increases depended neither upon conditions of blood $\mathrm{pH}$ or carbon dioxide tension which were variously changed by controlling respiration, nor upon systemic blood pressure changes.

Right internal carotid blood flow changes after stellate ganglion block was measured in a 60 years old man who had bilateral huge parasagittal meningioma. Before stellate ganglion block internal carotid flow was $377.6 \mathrm{ml} / \mathrm{m}$, began to 
increase 3 minutes later and reached the maximum $400.0 \mathrm{ml} / \mathrm{m}$ within 5 minutes which lasted the following ten minutes of observation.

In conclusion, it seems justifiable to say that the cerebral circulation is controlled to some extent by cervical sympathetic system and stellate ganglion block may afford good opportunity to regain its normal function for the anoxic brains.

\title{
k-22. Physiological and Clinical Findings of Some Particular Symptoms which are Discernible in Traumatic Cervical Syndrome
}

\author{
Naoki Kageyama, Mamoru Tanaka, Kentaro Koshino, Kimiyuki IKeda, \\ Morimasa Kohno, Shoji NaKaJima, Satoru Kubota, Tadahisa Kurimoto, \\ Yasuhiro TsuJi and Takayuki NAKAJIMA \\ Department of Neurosurgery, Kansai Medical School \\ Tokuji Nogawa \\ Department of Neurosurgery, Nogawa Hospital
}

Various kinds of symptoms are recognized in the patients who have been suffering from traumatic cervical syndrome. Some of them, such as finger tremor and dental symptoms, have not been watched with keen interest. We'll report here some physiological and clinical findings about them.

Finger tremors were observed in 73 cases of 260 patients of traumatic cervical syndrome and dental signs were seen in 58 cases. The analysis of frequency distribution of the finger tremor displays the bimodal frequency pattern with dominance at about $9 \mathrm{c} / \mathrm{sec}$. and $15-20 \mathrm{c} / \mathrm{sec}$. The energy of the finger tremor which is computed from the frequency distribution pattern through the frequency analyser, remarkably reduces in parallel with the relief of the pain with the treatment of xylocaine injection of the stellate ganglion or the great occipital nerve. This fact suggests that the physiological finger tremors are potentiated by the pain in case of the traumatic cervical syndrome.

It has been studied that the stimulation of the upper cervical sympathetic nerves effects upon the vasodilatation in the oral region, which causes the pain in the lower jaw on the basis of stimulation of vasomotor fibers rather than ascribing it to direct stimulation of afferent fibers in the sympathetic nerves. We studied the effects of the stimulation of the upper cervical autonomic nerves on the gingival temperature of the lower jaw, which, as was mentioned by Hamada (1965), is an indicator of the blood flow in the same locus. The temporary increment of the temperature was constantly observed by the electrical stimulation. This temporary effects might gradually last for a long period in case of the inflammatory tooth which pre-exists the accident. 